\title{
Joint PP and PS AVO inversion based on Zoeppritz equations*
}

\author{
Xiucheng Wei ${ }^{1,2, *}$ and Tiansheng Chen ${ }^{1,2}$ \\ 1 SINOPEC Key Laboratory of Seismic Multi-Component Technique, Beijing 100083, China \\ ${ }^{2}$ Petroleum Exploration and Production Research Institute, SINOPEC, Beijing 100083, China
}

\begin{abstract}
Considering Zoeppritz equations, reflections of PP and PS are only the function of ratios of density and velocity. So the inversion results will be the same if the ratios are the same but values of density, velocities of $\mathrm{P}$ wave and S-wave are different without strict constraint. This paper makes efforts to explore nonlinear simultaneous PP and PS inversion with expectation to reduce the ambiguity of AVO analysis by utilizing the redundancy of multi-component AVO measurements. Accurate estimation of ratio parameters depends on independence of input data. There are only two independent AVO attributes for PP reflectivity (i.e. intercept and gradient) and two for PS reflectivity (i.e. pseudo-intercept and pseudo-gradient or extreme amplitude), respectively. For individual PP and PS inversion, the values of least-squares objective function do not converge around a large neighborhood of chosen true model parameters. Fortunately for joint PP and PS inversion the values of the least-squares objective function show closed contours with single minima. Finally the power function fitting is used to provide a higher precision AVO attributes than traditional polynomial fitting. By using the four independent fitting attributes (two independent attributes for PP and PS respectively), the inversion of four ratio parameters (velocities and densities) would be estimated with less errors than that in traditional method.
\end{abstract}

Key words: multi-components; AVO; simultaneous inversion

CLC number: P315.3 Document code: A

\section{Introduction}

AVO analysis and inversion techniques have been used throughout the world in reservoir prediction. In the attempt to understand subsurface lithology, it is useful to jointly apply the properties of PP and PS AVO (Miles and Gassaway, 1989). Smith and Gidlow (1987) outlined a least-squares weighted-stacking procedure incorporating PP seismic data to extract compressional and shear velocities. This method utilizes NMO corrected pre-stack PP seismic data. Ferguson and Stewart (1996) described a similar method to derive estimates of shear velocity directly from an NMO corrected CCP gather. Both of the methods utilize a background velocity depth model to compute incidence and reflection angles. The primary disadvantage of the PS method is

\footnotetext{
* Received 7 July 2011; accepted in revised form 24 July 2011; published 10 August 2011.

+ Corresponding author. e-mail: weixc.syky@sinopec.com

(c) The Seismological Society of China and Springer-Verlag Berlin Heidelberg 2011
}

the need for an additional background density model. A true simultaneous method first given by Stewart (1990) outlines a procedure that incorporates both PP and PS seismic gathers in a joint PP and PS inversion. One problem inherent with the Smith and Gidlow approach was the incorporation of Gardner's relation, which may not be applicable sometimes. This method was further developed by Fatti et al. (1994) to eliminate this dependency upon Gardner's relation to estimate density. Smith (1996) and Lines (1998) extended the weighted-stacking method to the case of three-parameter inversion. The three elastic parameters would be either relative changes in $\mathrm{P}$-wave velocity $(\Delta \alpha / \alpha)$, S-wave velocity $(\Delta \beta / \beta)$ and density $(\Delta \rho / \rho)$ or P-wave impedance $\left(\Delta I_{\mathrm{P}} / I_{\mathrm{P}}\right)$, S-wave impedance $\left(\Delta I_{\mathrm{S}} / I_{\mathrm{S}}\right)$. Drufuca and Mazzotti (1995) and Debski and Tarantola (1995) showed the potential ambiguities inherent with the three-parameter inversion. Lines (1998) further showed that density determination is difficult for limited apertures (limited incidence angles) and typical seismic velocities. The application of PS seismic data 
to this problem should further constrain the three parameter solution and thus allow for more reasonable acquisition parameters to be used. The weighted-stacking technique of simultaneous PP and PS AVO inversion is based on the first-order Zoeppritz equations approximations of PP and PS reflectivity respectively, which has high accuracy within small incident angles (about less than 30 degrees). Notice the PP Zoeppritz equations approximation accuracy is significantly higher than the PS approximation. Higher-order approximation may also be used to improve the accuracy of both PP and PS reflectivity, but is impractical due to the increased number of terms in the equations and the nonlinear

$$
\left[\begin{array}{ccc}
-\sin \theta_{1} & -\cos \varphi_{1} & \sin \theta_{2} \\
\cos \theta_{1} & -\sin \varphi_{1} & \cos \theta_{2} \\
\sin 2 \theta_{1} & \frac{\alpha_{1}}{\beta_{1}} \cos 2 \varphi_{1} & \frac{\rho_{2}}{\rho_{1}} \frac{\alpha_{1}}{\alpha_{2}} \frac{\beta_{2}^{2}}{\alpha_{1}^{2}} \frac{\alpha_{1}^{2}}{\beta_{1}^{2}} \sin 2 \theta_{2} \\
-\cos 2 \varphi_{1} & \frac{\beta_{1}}{\alpha_{1}} \sin 2 \varphi_{1} & \frac{\rho_{2}}{\rho_{1}} \frac{\alpha_{2}}{\alpha_{1}} \cos 2 \varphi_{2}
\end{array}\right.
$$

where $\alpha_{1}, \alpha_{2}, \beta_{1}, \beta_{2}, \rho_{1}, \rho_{2}$ are separately the P-wave velocities, $\mathrm{S}$-wave velocities and densities across an interface, $\theta_{1}$ and $\theta_{2}$ are the incidence and transmission angle of $\mathrm{P}$-wave across the interface, and $\varphi_{1}$ and $\varphi_{2}$ are the reflection and transmission angles of S-wave across the interface. $R_{\mathrm{PP}}$ and $R_{\mathrm{PS}}$ are PP and PS reflection coefficients respectively. $T_{\mathrm{PP}}$ and $T_{\mathrm{PS}}$ are $\mathrm{PP}$ and PS transmission coefficients respectively.

The equations are nonlinear functions with respect to the six elastic parameters, that is $\alpha_{1}, \alpha_{2}, \beta_{1}, \beta_{2}, \rho_{1}, \rho_{2}$. The ultimate goal of an AVO inversion method is to find the six elastic parameters input to the Zoeppritz equations. Generalized linear inversion (GLI) has been applied extensively in the solution of AVO inversion problems (Larsen, 1999). The uniqueness of a solution to AVO inversion is dependent upon the selection of the parameters to be inverted. Following Larsen (1999), since densities in the Zoeppritz equations appear in the form of ratios, the following vector was chosen as inversion parameters:

$$
\boldsymbol{P}=\left[\alpha_{1}, \alpha_{2}, \beta_{1}, \beta_{2}, \rho_{2} / \rho_{1}\right] .
$$

According to two-parameter linear approximations of PP or PS, it is found that there are only two independent AVO attributes for PP or PS reflection coefficient respectively. So individual $\mathrm{PP}$ and $\mathrm{PS}$ AVO inversions are only suitable to invert two parameters steadily; while joint PP and PS AVO inversion is suitable to dependence on the velocity and density contrasts. Larsen (1999) introduced the generalized linear inversion method to simultaneously invert the exact Zoeppritz equations solution for PP and PS reflectivity and improved the accuracy for elastic parameters estimates.

\section{Nonlinear simultaneous PP and PS inversion}

The Zoeppritz equations (1) fully describe the relationship between angles, reflected and transmitted coefficients of compressional and shear waves on either side of a plane interface.

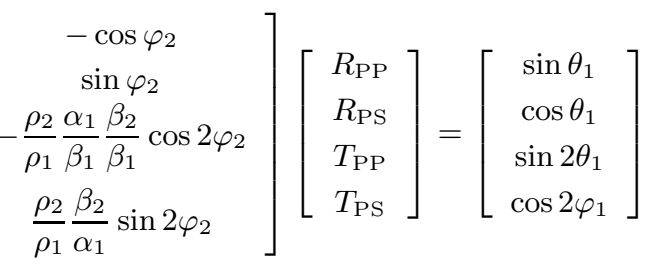

invert four parameters steadily. There are five unknown parameters in equations (1), so joint inversion is not enough to solve the elastic parameters steadily.

It is well known that reflection coefficients of PP and PS are the integrated influence of elastic parameters across the interface. Given two models with same ratio parameters $\left(\rho_{2} / \rho_{1}, \alpha_{2} / \alpha_{1}, \beta_{1} / \alpha_{1}, \beta_{2} / \alpha_{1}\right)$, but different elastic parameters $\left(\alpha_{1}, \alpha_{2}, \beta_{1}, \beta_{2}, \rho_{1}, \rho_{2}\right)$, their AVO responses are same. Therefore, the model parameters $\left(\alpha_{1}, \alpha_{2}, \beta_{1}, \beta_{2}, \rho_{1}, \rho_{2}\right)$ are unavailable without strict constraint. From equations (1), the PP and PS reflection coefficients can be simply expressed as the function of four independent ratio parameters as follows:

$$
\boldsymbol{P}=\left[\frac{\rho_{2}}{\rho_{1}}, \frac{\alpha_{2}}{\alpha_{1}}, \frac{\beta_{1}}{\alpha_{1}}, \frac{\beta_{2}}{\alpha_{1}}\right] .
$$

Following the least squares error theory, the object function of nonlinear inversion of the Zoeppritz equations is residual error function. For the case of joint PP and PS inversion, the objective function is written as

$$
\begin{aligned}
\varepsilon_{\mathrm{PP}+\mathrm{PS}}(P)= & \sum_{i=1}^{N}\left[R_{\mathrm{PP}, i}-R_{\mathrm{PP}, i}^{\mathrm{M}}(P)\right]^{2}+ \\
& \sum_{i=1}^{N}\left[R_{\mathrm{PS}, i}-R_{\mathrm{PS}, i}^{\mathrm{M}}(P)\right]^{2},
\end{aligned}
$$

where $R_{\mathrm{PP}, i}^{\mathrm{M}}(P)$ and $R_{\mathrm{PS}, i}^{\mathrm{M}}(P)$ are the forward model response for a given set of model parameter vectors, i.e., 
the PP and PS reflection coefficient Zoeppritz equation solution respectively. $R_{\mathrm{PP}, i}(P)$ and $R_{\mathrm{PS}, i}(P)$ are the real seismic record information (observations) of $\mathrm{PP}$ and PS, respectively. Equation (4) is evaluated for all offsets (incident angles) from 1 to $N$, where $N$ is the total number of offsets.

For incidence angles up to 30 degrees and small contrasts in elastic properties, PP and PS AVO equations can be simplified to linear approximations with only two coefficients (Ramos and Castagna, 2001); the usual AVO attributes are intercept and gradient for PP wave and pseudo-intercept and pseudo-gradient for PS wave. If AVO attributes are selected to express reflection coefficients, equations (4) can be written as

$$
\begin{aligned}
\varepsilon_{\mathrm{PP}+\mathrm{PS}}(P)= & \left(A_{\mathrm{PP}}^{\mathrm{O}}-A_{\mathrm{PP}}^{\mathrm{M}}\right)^{2}+\left(B_{\mathrm{PP}}^{\mathrm{O}}-B_{\mathrm{PP}}^{\mathrm{M}}\right)^{2}+ \\
& \left(A_{\mathrm{PS}}^{\mathrm{O}}-A_{\mathrm{PS}}^{\mathrm{M}}\right)^{2}+\left(B_{\mathrm{PS}}^{\mathrm{O}}-B_{\mathrm{PS}}^{\mathrm{M}}\right)^{2},
\end{aligned}
$$

where $A_{\mathrm{PP}}$ and $B_{\mathrm{PP}}$ denote intercept and gradient for $\mathrm{PP}$ wave, $A_{\mathrm{PS}}$ and $B_{\mathrm{PS}}$ denote pseudo-intercept and pseudo-gradient for PS wave. Superscripts ' $\mathrm{O}$ ' and ' $\mathrm{M}$ ' represent observational and modeling AVO attributes respectively. The linear PS approximation with two coefficients (Ramos and Castagna, 2001) only satisfies the inversion precision at small incidence angles at which the signal to noise ratio is lower for converted waves. At middle to far offsets the converted wave amplitude reaches an extreme and the signal to noise ratio is higher. Extreme amplitude attribute (Chen and Wei, 2007; Wei et al., 2008) is a better PS AVO attribute instead of pseudo-gradient in equation (5).

Once model parameter vector $\boldsymbol{P}$ is inverted, some other attributes can be directly calculated from estimates of each element. The fractional changes in density and velocity can be formulated as

$$
\left\{\begin{array}{l}
\frac{\Delta \rho}{\rho}=2 \frac{P_{1}-1}{P_{1}+1} \\
\frac{\Delta \alpha}{\alpha}=2 \frac{P_{2}-1}{P_{2}+1} \\
\frac{\Delta \beta}{\beta}=2 \frac{P_{4} / P_{3}-1}{P_{4} / P_{3}+1}
\end{array}\right.
$$

where $P_{i}(i=1,2,3,4)$ denotes the element of model parameter vector. The fractional changes in impedance can be expressed as

$$
\frac{\Delta I}{I}=2 \frac{P_{1} P_{2}-1}{P_{1} P_{2}+1} \quad \text { and } \quad \frac{\Delta J}{J}=2 \frac{P_{1} P_{4} / P_{3}-1}{P_{1} P_{4} / P_{3}+1} .
$$

The fractional changes in Lame parameters can be expressed as

$$
\frac{\Delta(\rho \lambda)}{\rho \lambda}=2 \frac{P_{1}^{2}\left(1-P_{3}^{2}\right)\left(P_{2}^{2}-P_{4}^{2}\right)-1}{P_{1}^{2}\left(1-P_{3}^{2}\right)\left(P_{2}^{2}-P_{4}^{2}\right)+1}
$$

$$
\frac{\Delta(\rho \mu)}{\rho \mu}=2 \frac{\left(\frac{P_{1} P_{4}}{P_{3}}\right)^{2}-1}{\left(\frac{P_{1} P_{4}}{P_{3}}\right)^{2}+1}
$$

and

$$
\frac{\Delta(\lambda / \mu)}{\lambda / \mu}=2 \frac{\frac{\left(1-P_{3}^{2}\right)\left(P_{2}^{2}-P_{4}^{2}\right)}{\left(P_{3} P_{4}\right)^{2}}-1}{\frac{\left(1-P_{3}^{2}\right)\left(P_{2}^{2}-P_{4}^{2}\right)}{\left(P_{3} P_{4}\right)^{2}}+1} .
$$

The fraction Poisson's ratio and bulk modulus ratio can also be defined as

$$
\frac{\Delta \sigma}{\sigma}=2 \frac{\frac{\left(2 P_{4}^{2}-P_{2}^{2}\right)\left(P_{3}^{2}-1\right)}{\left(P_{4}^{2}-P_{2}^{2}\right)\left(2 P_{3}^{2}-1\right)}-1}{\frac{\left(2 P_{4}^{2}-P_{2}^{2}\right)\left(P_{3}^{2}-1\right)}{\left(P_{4}^{2}-P_{2}^{2}\right)\left(2 P_{3}^{2}-1\right)}+1}
$$

and

$$
\frac{\Delta(\kappa \rho)}{\kappa \rho}=2 \frac{P_{1}^{2}\left(P_{2}^{2}-4 / 3 P_{4}^{2}\right) /\left(1-4 / 3 P_{3}^{2}\right)-1}{P_{1}^{2}\left(P_{2}^{2}-4 / 3 P_{4}^{2}\right) /\left(1-4 / 3 P_{3}^{2}\right)+1} .
$$

The two term linear approximations (Ramos and Castagna, 2001) of PP and PS can be simplified to

$$
\left\{\begin{array}{ll}
y=a_{\mathrm{PP}} x^{2}+c_{\mathrm{PP}} & \text { (for PP wave) } \\
y=a_{\mathrm{PS}} x^{3}+c_{\mathrm{PS}} x & \text { (for PS wave) }
\end{array} .\right.
$$

Because the linear approximations of PP and PS is the first order equation of Taylor series, equation (13) can be generalized to (Wei et al., 2008):

$$
\begin{cases}y=a_{\mathrm{PP}} x^{b_{\mathrm{PP}}}+c_{\mathrm{PP}} & \text { (for PP wave) } \\ y=a_{\mathrm{PS}} x^{b_{\mathrm{PS}}}+c_{\mathrm{PS}} x & \text { (for PS wave) }\end{cases}
$$

where coefficient $b$ is variable, so equation (14) includes effects caused by higher-order terms and improves the fitting precision at middle and far offsets.

\section{Examples}

The following set of model parameters (Table 1) was used to test the accuracy of simultaneous PP and PS AVO inversion. Model parameter vector is $\boldsymbol{P}=[0.9345,1.111$ 9, 0.548 7, 0.844 8]. Figure 1 shows the PP and PS AVO responses to the model. PP AVO

Table 1 Model parameters

\begin{tabular}{cccc}
\hline & $\alpha /\left(\mathrm{km} \cdot \mathrm{s}^{-1}\right)$ & $\beta /\left(\mathrm{km} \cdot \mathrm{s}^{-1}\right)$ & $\rho /\left(\mathrm{g} \cdot \mathrm{cm}^{-3}\right)$ \\
\hline Shale & 2.7700 & 1.5200 & 2.2900 \\
Gas sand & 3.0800 & 2.3400 & 2.1400 \\
\hline
\end{tabular}




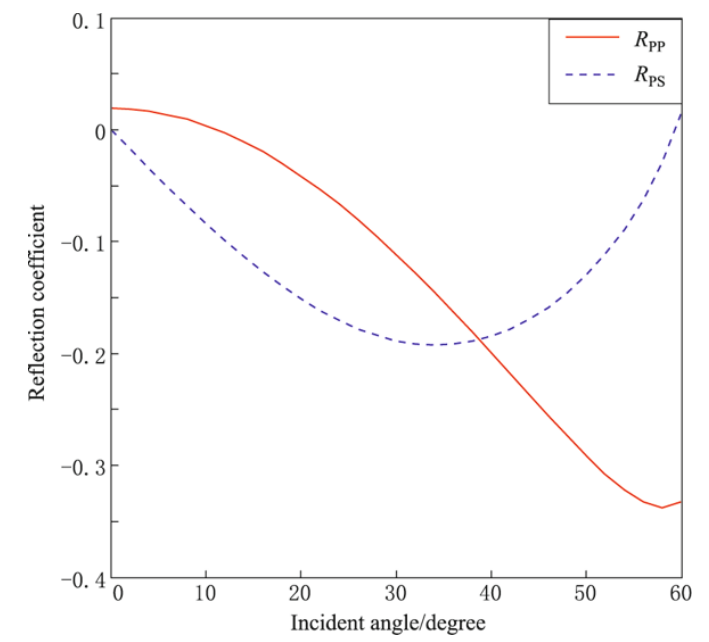

Figure $1 \quad \mathrm{PP}$ and PS AVO responses to model (Table 1).

response is almost linear at small incident angle $\left(<30^{\circ}\right)$, whereas the PS AVO response reach an extreme at middle incident angel. To each comparable inversion results, four incidence angles $\left(5^{\circ}, 10^{\circ}, 15^{\circ}\right.$ and $20^{\circ}$ ) are chosen for $\mathrm{PP}$ inversion alone and four incident angles $\left(10^{\circ}, 25^{\circ}, 35^{\circ}\right.$ and $\left.45^{\circ}\right)$ for PS inversion alone. Specially two PP data (such as incident angles $10^{\circ}$ and $20^{\circ}$ ) and two PS data (such as incident angles $35^{\circ}$ and $45^{\circ}$ ) are chosen for joint PP and PS inversion. To test the convergence of object function around a large neighborhood of chosen model parameters, given parameters $\rho_{2} / \rho_{1}$ and $\alpha_{2} / \alpha_{1}$ are the value of model parameter 0.9345 and 1.1119 respectively; parameter $\beta_{1} / \alpha_{1}$ ranges from 0.2 to 1.0 (0.3 to 0.8 displayed in Figure 2 ) and $\beta_{2} / \alpha_{1}$ ranges from 0.3 to 1.5 (0.5 to 1.0 displayed in Figure 2). The values of objective function are used to investigate the degree of convergence (Figure 2). In the case of PP and PS inversion alone, the values of objective function show closed contours with multi-minima, whereas in the case of joint PP and PS inversion only a single minimum. These indicate that nonlinear PP or PS inversion alone easily fall into local solution that depends on initial parameters, while the joint PP and PS inversion anyway search for optimized solution (Table 2). At small incident angle $\left(<30^{\circ}\right)$, linear AVO response of PP and PS can only provides two independent attributes respectively (gradient and intercept for PP reflectivity, pseudo-gradient and pseudo-intercept for PS reflectivity). At middle

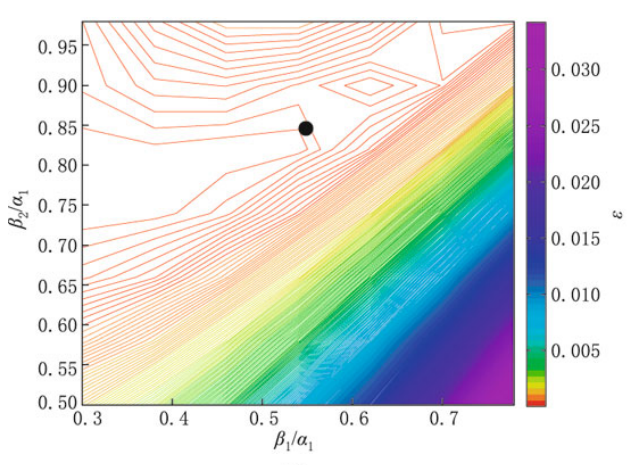

(a)

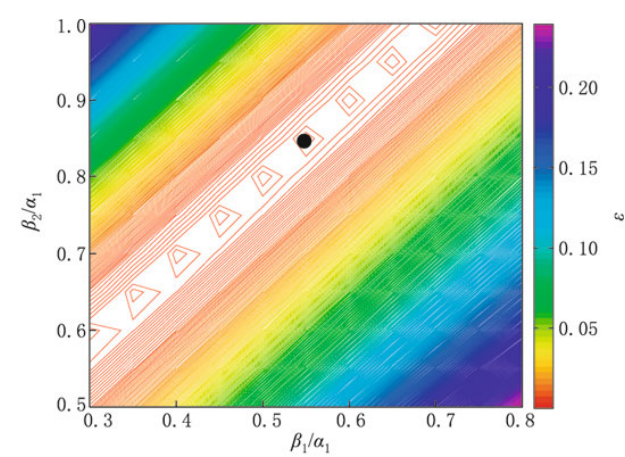

(b)

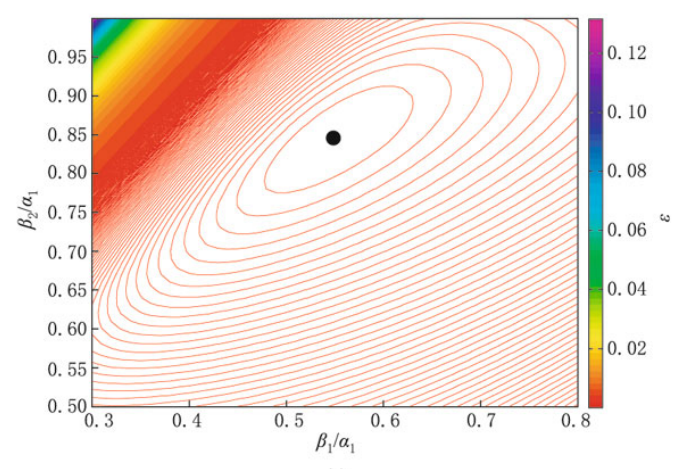

(c)

Figure 2 Comparison of the values of objective function $\varepsilon$ between PP alone (a), PS alone (b) and joint PP and PS inversions (c). The parameters $\rho_{2} / \rho_{1}$ and $\alpha_{2} / \alpha_{1}$ are true value 0.9345 and 1.1119 respectively, parameter $\beta_{1} / \alpha_{1}$ ranges from 0.3 to 0.8 and $\beta_{2} / \alpha_{1}$ ranges from 0.5 to 1.0. Exact values are denoted by black dot. 

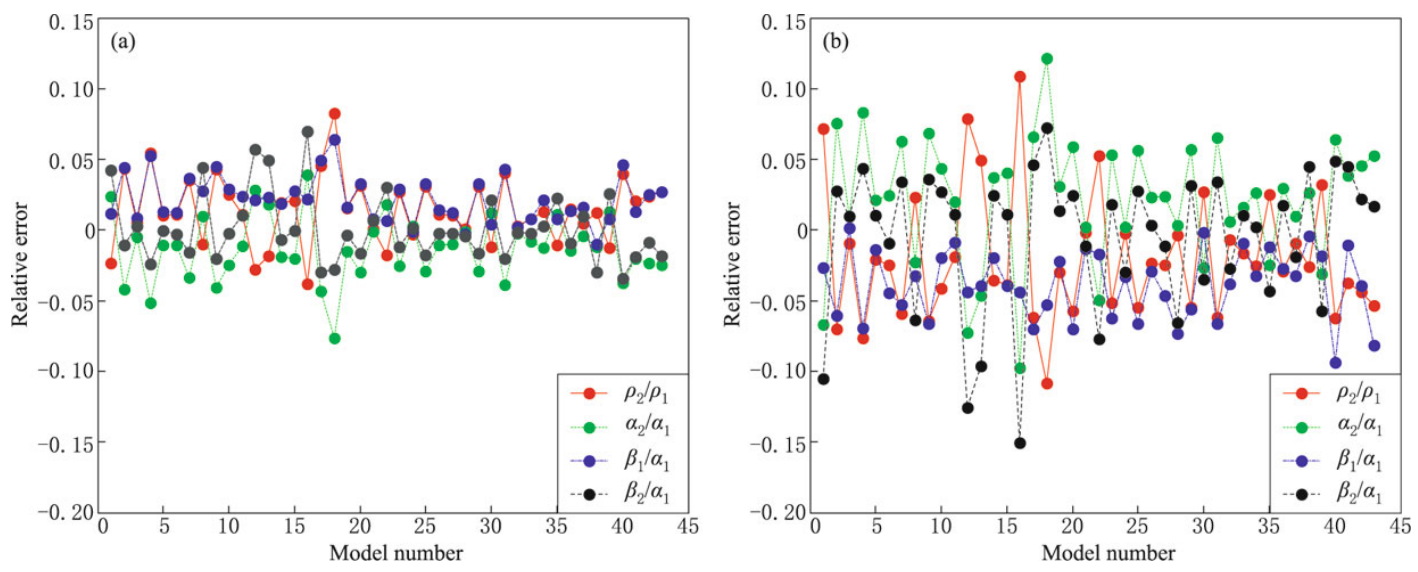

Figure 3 The fractional error of model parameters obtained from joint PP and PS inversion. Four input parameters (two from PP and two from PS respectively) are obtained from power fits (a) and polynomial fits (b) to AVO curves.

Table 2 Inversion of model parameters vector

\begin{tabular}{ccccc}
\hline & $\rho_{2} / \rho_{1}$ & $\alpha_{2} / \alpha_{1}$ & $\beta_{1} / \alpha_{1}$ & $\beta_{2} / \alpha_{1}$ \\
\hline Exact & 0.9345 & 1.1119 & 0.5487 & 0.8448 \\
PP inversion & 0.6538 & 1.5906 & 0.8636 & 1.3304 \\
PS inversion & 0.4018 & 0.2421 & 0.6585 & 1.2467 \\
PP and PS inversion & 0.9345 & 1.1119 & 0.5487 & 0.8447 \\
\hline
\end{tabular}

offsets, the converted wave amplitude reaches an extreme with high $\mathrm{S} / \mathrm{N}$ ratio. Converted wave AVO curve fits using the power function presented here has a higher precision than the third-order polynomial fits (Wei et al., 2008), so power function fits is expanded to fit PP and PS AVO amplitude at middle offsets and then four high precision independent attributes are provided for joint AVO inversion. Figure 3 shows the fractional error of model parameters obtained from joint PP and PS inversion. The inversion accuracy with power functions [equation (14)] is significant better than that with polynomial functions [equation (13)].

\section{Conclusions}

This paper presented a practical method for joint inversion of PP and PS reflection data, which expresses Zoeppritz equations as functions of four independent ratio parameters $\boldsymbol{P}=\left[\rho_{2} / \rho_{1}, \alpha_{2} / \alpha_{1}, \beta_{1} / \alpha_{1}, \beta_{2} / \alpha_{1}\right]$. The values of objective function of $\mathrm{PP}$ or $\mathrm{PS}$ inversion alone show closed contours with multi-minima, and it is difficult to converge to true model parameters; whereas that of joint PP and PS inversion shows closed contours with a single minimum around a large neighborhood of the true model parameters, so it is easy to search for the global optimization solution steadily. The power func- tion fitting presented provides a higher precision AVO attributes than traditional polynomial fitting. By using four independent fitting attributes (two for PP and two for PS respectively), this study draws a conclusion that better estimates on ratio of elastic parameters are obtained from simultaneous PP and PS inversion. Once model parameters vector $\boldsymbol{P}$ is inverted, some other attributes can be directly calculated, such as the fractional changes in density, velocity, impedance, Lame parameters, Poisson's ratio and bulk modulus ratio.

Acknowledgements This work was funded by National 973 Basic Research Developments Program of China (No. 2005CB422104), 863 National High Technique Research Development Project of China (No. 2007AA060505) and National Natural Science Foundation of China (No. 40839901).

\section{References}

Chen T S and Wei X C (2007). Inversion of velocity ratio $v_{\mathrm{P}} / v_{\mathrm{S}}$ from converted wave AVO. 76th Ann. Internat. Mtg., Soc. Expl. Geophys., Expanded Abstracts, 9991002.

Debski W and Tarantola A (1995). Information on elastic parameters obtained from the amplitudes of reflected waves. Geophysics 60: 1 426-1 436.

Drufuca G and Mazzotti A (1995). Ambiguities in AVO inversion of reflections from a gas-sand. Geophysicss $\mathbf{6 0}$ : $134-141$.

Fatti J L, Smith G C, Vail P J, Strauss P J and Levitt P $R$ (1994). Detection of gas in sandstone reservoirs using AVO analysis: A 3-D seismic case history using the Geostack technique. Geophysics 59(6): 1 362-1 376.

Ferguson R J and Stewart R R (1996). Shear Wave Interval Velocity From P-S Stacking Velocities. CREWES Re- 
search Report, The University of Calgary, Vol. 8, Chapter $22,1-7$.

Larsen J A (1999). AVO Inversion by Simultaneous $P-P$ and $P-S$ Inversion. [M.S. Dissertation]. Department of Geology and Geophysics, The University of Calgary, 28-35.

Lines L R (1998). Density Contrast is Difficult to Determine From AVO. CREWES Research Report, The University of Calgary, Vol. 10, Chapter 47, 1-8.

Miles D R and Gassaway G S (1989). Three-component AVO analysis. 59th Ann. Internat. Mtg., Soc. Expl. Geophys., Expanded Abstracts, 706-708.

Ramos A B C and Castagna J P (2001). Useful approximations for converted-wave AVO. Geophysics 66(6): 1 7211734 .
Smith G C (1996). 3-parameter geostack. 66th Annual Internat. Mtg., Soc. Expl. Geophys., Expanded Abstract, 1 7471750.

Smith G C and Gidlow P M (1987). Weighted stacking for rock property estimation and detection of gas. Geophysical Prospecting 35: 993-1 014.

Stewart R R (1990). Joint $P$ and P-SV Inversion. CREWES Research Report, The University of Calgary, Vol. 3, 112115.

Wei S, Li X Y, Chen T and Ji Y (2008). Converted wave AVO inversion for average velocity ratio and shear wave reflection coefficient. 78th Ann. Internat. Mtg., Soc. Expl. Geophys., Expanded Abstracts, 269-272. 